\title{
Simulation Study on Kinetic Parameter Estimation Using Artificial Neural Networks
}

\author{
Kenya Murase ${ }^{\dagger}$, Shuji TANADA and Junpei IKEZOE \\ Department of Radiology, Ehime University School of Medicine \\ Shitsukawa, Shigenobu-cho, Onsen-gun, Ehime Pref. 791-02, Japan
}

Received March 3, 1997

\begin{abstract}
We applied artificial neural networks (ANNs) to kinetic parameter estimation and investigated their performance using a computer-based simulation method. The ANNs used in the present study were based on a feed-forward layered model with an input, hidden, and output layers, on which a backpropagation learning model was implemented. In the computer simulation, the three-compartment fluorodeoxyglucose model was used. The brain time-activity data were generated using the mean and standard deviation of the rate constants in the literature and the plasma time-activity data obtained from actual positron emission tomography studies. These time-activity data and the rate constants were used as input and output data for training ANNs, respectively. The performance of ANNs was generally better with increasing number of hidden nodes and training samples. Although the accuracy of the parameter estimates obtained by ANNs was lower or comparable to that obtained by the nonlinear least-squares (NLSQ) method at lower noise levels, it was generally better than that obtained by the NLSQ method at higher noise levels. Although the computational cost for training ANNs was very high, ANNs could estimate the parameters very fast once they were trained adequately. These results suggested that an ANN approach may be an effective strategy for kinetic parameter estimation.
\end{abstract}

Key Words: artificial neural network, kinetic parameter estimation, simulation study, backpropagation, fluorodeoxyglucose model

\section{Introduction}

Artificial neural networks (ANNs) are a computational model based on the human brain, and are a powerful tool for pattern recognition ${ }^{1), 2}$. In recent years, ANN has been applied to a broad range of problems in the field of medicine ${ }^{3)}$.

Kinetic parameter estimation using positron emission tomography (PET) or single photon emission computed tomography (SPECT) is usually performed using the nonlinear least-squares

${ }^{\dagger}$ All correspondence to Kenya Murase, PhD, Department of Radiology, Ehime University School of Medicine, Shitsukawa, Shigenobucho, Onsen-gun, Ehime Pref. 791-02, Japan. Tel : 089-960-5371; Fax : 089-960-5375; e-mail : murase@dpc.ehime-u.ac.jp
(NLSQ) method ${ }^{4)-6)}$, the graphical analysis technique $^{7,8)}$, and the weighted-integration method ${ }^{9}$. Although the NLSQ method is usually used as a standard, it is not practical for deriving pixel-bypixel parametric images because of its computational inefficiency. In contrast, the graphical analysis technique and the weighted-integration method are applicable to generation of parametric images because of their computational efficiency. In the case of ANN, once it is trained adequately, it can estimate the parameters very fast since the output is generated in a single pass without repeated iterations or complicated procedures. For this respect, there is a possibility that an ANN approach can be applied to generation of parametric images of kinetic parameters. The purpose of 
the present study was to investigate the applicability of ANNs to kinetic parameter estimation, using the computer-based simulation method.

\section{Materials and Methods}

\subsection{Artificial neural networks}

ANNs consist of simple processing units called "neurons" or "nodes". The software of ANNs used is based on a feed-forward layered model with an input layer, a hidden layer, and an output layer as illustrated in Fig. 1, on which a so-called backpropagation learning model ${ }^{10)}$ with a Levenberg-Marquardt optimizer ${ }^{11}$ is implemented.

The output data are provided from output units through two successive nonlinear calculations in the hidden and output layers. The calculations at each unit in a layer include weighted summation of all entry numbers (weight) and addition of a certain offset number (bias), by using a sigmoidshaped function (log-sigmoid) ${ }^{1}$. A training signal is given to the ANN, and the backpropagation technique modifies weight and bias values so that the summation of the squared errors in the output layer is minimized. This modification of the

\section{Input layer Hidden layer Output layer}

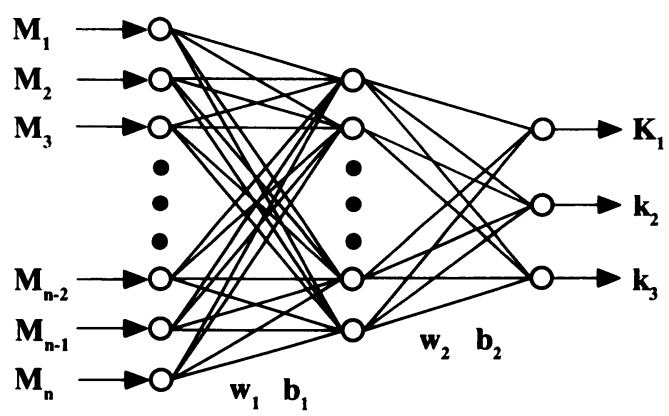

Fig. 1 Illustration of the artificial neural network (ANN) used in the present study.

$w_{1}$ and $b_{1}$ represent the weight and bias between the input and hidden layers, while $w_{2}$ and $b_{2}$ represent the weight and bias between the hidden and output layers, respectively. weight and bias values is called a learning or training process.

\section{$2 \cdot 2$ Simulation study}

In the present simulation study, we used the 3-compartment fluorodeoxyglucose (FDG) model ${ }^{4), 5)}$, in which the dephosphorylation of FDG-6-phosphate to FDG and the radioactivity attributable to the vascular compartment were assumed to be negligible for simplicity. With this model, the radioactivity measured during the $i$-th scan can be expressed by

$$
M_{i}=\frac{\int_{T_{i-1}}^{T_{i}} M(t) \mathrm{d} t}{T_{i}-T_{i-1}},
$$

where $T_{i-1}$ and $T_{i}$ are the start and end times of the $i$-th scan, respectively, and $M(t)$ is given by ${ }^{4), 5)}$

$$
\begin{aligned}
M(t)= & K_{1} \frac{k_{3}}{k_{2}+k_{3}} \int_{0}^{t} C_{a}(u) \mathrm{d} u \\
& +K_{1} \frac{k_{2}}{k_{2}+k_{3}} \int_{0}^{t} C_{a}(u) \mathrm{e}^{-\left(k_{2}+k_{3}\right)(t-u)} \mathrm{d} u .
\end{aligned}
$$

$K_{1}, k_{2}$, and $k_{3}$ in the above equation represent the unidirectional clearance of FDG from blood to brain, the fractional clearance of FDG from brain to blood, and the phosphorylation coefficient of FDG to FDG-6-phosphate, respectively, and $C_{\mathrm{a}}(t)$ is the arterial plasma FDG concentration.

"Noiseless" brain time - activity data were generated from a set of $K_{1}, k_{2}, k_{3}$, and the plasma time - activity data from actual PET studies illustrated in Fig. 2 (a) ${ }^{12)}$, using Eq.(1). For generation of the brain time - activity data, the values of mean and standard deviation (SD) of $K_{1}, k_{2}$, and $k_{3}$ were obtained from the literature $\mathrm{e}^{4), 5), 13), 14}$ and then a set of these rate constants was generated using the mean and SD values as follows :

$$
\begin{aligned}
K_{1} & =0.0900+\operatorname{rand} n \times 0.0195(\mathrm{ml} / \mathrm{g} / \mathrm{min}) \\
k_{2} & =0.1502+\operatorname{rand} n \times 0.0432\left(\mathrm{~min}^{-1}\right), \quad(3) \\
k_{3} & =0.0814+\operatorname{rand} n \times 0.0277\left(\mathrm{~min}^{-1}\right)
\end{aligned}
$$


where randn is a normally distributed random number with a mean of zero and SD of unity. The $M_{i}$ values given by Eq.(1) were generated from the above rate constants over a set of intervals using the following scanning sequence : six 30-s, seven 1-min, five 2-min, and ten 5-min scans ${ }^{12}$. In order to investigate the effect of the statistical noise, random noise was added to the "noiseless" time - activity data using a Poisson distribution of mean zero and SD $\sigma_{i}$ given by ${ }^{15)}$

$$
\frac{\sigma_{i}}{N_{i}}=\frac{F}{100} \sqrt{\frac{N_{\mathrm{m}}}{N_{i}}},
$$

where $N_{i}$ is the total counts in scan $i, N_{\mathrm{m}}$ the mean count per scan, and $F$ the noise level (\%) for mean count. Examples of the radioactivities calculated from Eq. (1) for three noise levels are shown in Figs. 2 (b), (c), and (d). Figures 2 (b), (c), and (d) show the cases when the noise level is $0 \%, 5 \%$, and $10 \%$, respectively. In this case, $K_{1}$, $k_{2}$, and $k_{3}$ were $0.1127 \mathrm{ml} / \mathrm{g} / \mathrm{min}, 0.1773 \mathrm{~min}^{-1}$, and $0.0835 \mathrm{~min}^{-1}$, respectively.

The brain time - activity data generated and the rate constants $K_{1}, k_{2}$, and $k_{3}$ were used as input and output data for training ANNs (Fig. 1), respectively. In the present study, the plasma time activity data was not taken into consideration in ANNs for simplicity. This corresponds to simulating the case when a standardized input function ${ }^{16)}$
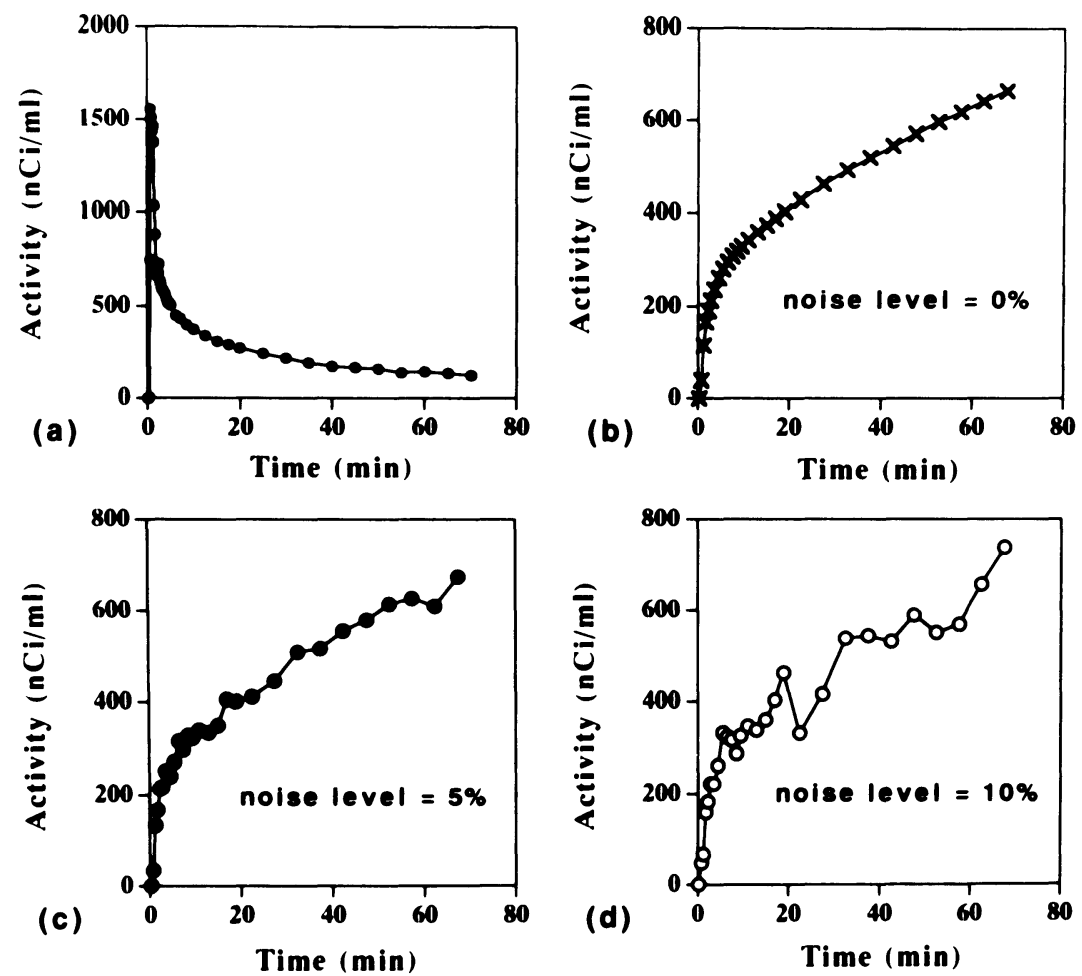

Fig. 2 (a) Plasma time-activity data from actual PET studies used in the present study. (b), (c), and (d) are the brain time-activity data generated from Eq. (1) using $0.1127 \mathrm{ml} / \mathrm{g} / \mathrm{min}$ for $K_{1}, 0.1773 \mathrm{~min}^{-1}$ for $k_{2}$, and $0.0835 \mathrm{~min}^{-1}$ for $k_{3}$, for three noise levels ((a) for $0 \%$, (b) for $5 \%$, and (c) for $10 \%)$.

These data represent examples of input data used for training ANNs in the present study. 
was used.

In addition to $K_{1}, k_{2}$, and $k_{3}$, we estimated the net clearance of FDG from blood to brain $(K)$, which is defined as ${ }^{4), 5)}$

$$
K=\frac{K_{1} \cdot k_{3}}{k_{2}+k_{3}} \text {. }
$$

\section{$2 \cdot 3$ ANN performance evaluation}

First, we investigated the effect of the number of hidden nodes on an ANN performance for three noise levels $(0 \%, 5 \%$, and $10 \%)$ using 500 training samples (Fig. 3). In this study, the number of hidden nodes was changed from 2 to 18 , and the number of learning iterations was fixed to 500 . We evaluated it in terms of both the correlation coefficients between the true and estimated values of $K_{1}, k_{2}$, and $k_{3}$ in the 500 training samples, and the summation of squared error (SSE) defined by

$$
\mathrm{SSE}=\sum_{i=1}^{N}\left(t_{i}-e_{i}\right)^{2},
$$

where $t_{i}$ and $e_{i}$ represent the true and estimated values of the $i$-th sample, respectively, and $N$ is the number of samples. In this case, $N$ is 500 .

Secondly, we examined the computation (cpu) time needed for training ANNs (Fig. 4). When investigating the relationship between the cpu time and the number of hidden nodes (Fig. 4 (a)), the number of training samples was fixed to 500. On the other hand, when investigating the relationship between the cpu time and the number of training samples (Fig. 4 (b)), the number of hidden nodes was fixed to 10 . In these cases, the number of learning iterations was fixed to 500 . Note that the cpu time was shown as the cpu time per iteration in Figs. 4 (a) and (b). Calculations were performed using $C$ language on Macintosh computer (PowerPC 8500/150, Apple Japan Inc.).

Finally, we examined the effect of the number of training samples on an ANN performance (Tables 1 and 2). To do this, we used 100, 500, 1000 , and 1500 samples for training, which were selected randomly from the brain time-activity data base. After training, each ANN was tested with 100 new randomly-selected samples. In this case, the number of hidden nodes was fixed to 10 . Each ANN was trained up to 1000 learning iterations or until SSE became less than $10^{-4}$. The accuracy of parameter estimation was evaluated in terms of root mean square error (RMSE), which is defined by

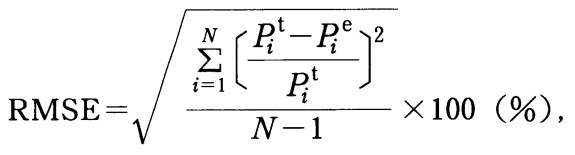

where $P_{i}{ }^{\mathrm{t}}$ and $\boldsymbol{P}_{i}{ }^{\mathrm{e}}$ are the true and estimated values of the $i$-th data of the parameter, respectively, and $N$ is the number of the data. The mean and SD values of the RMSE given by Eq.(7) were calculated for ten cases, with each consisting of 100 test samples (Table 1). The mean and SD values of the correlation coefficients between the true and estimated values were also calculated for ten cases, with each consisting of 100 test samples (Table 2). Comparison with the results obtained by the NLSQ method ${ }^{6)}$ was also performed to validate the present method.

\section{$2 \cdot 4$ Statistical analysis}

Statistical differences in RMSE (Table 1) and the correlation coefficients (Table 2) between ANNs and the NLSQ method were analyzed using the Mann-Whitney's U test. A p value less than 0.05 was considered significant.

\section{Results}

Figure 3 shows the simulation results in which the effect of the number of hidden nodes was investigated using 500 training samples. Figure 3 


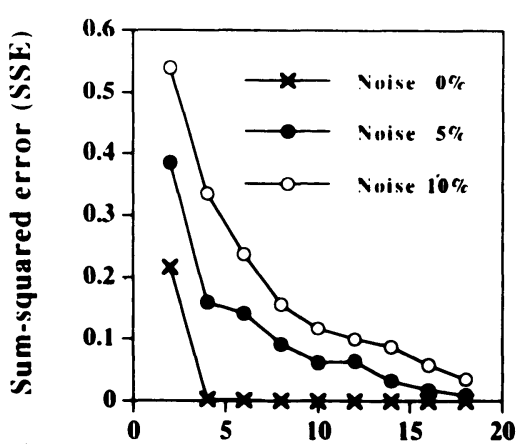

(a)

No. of hidden nodes

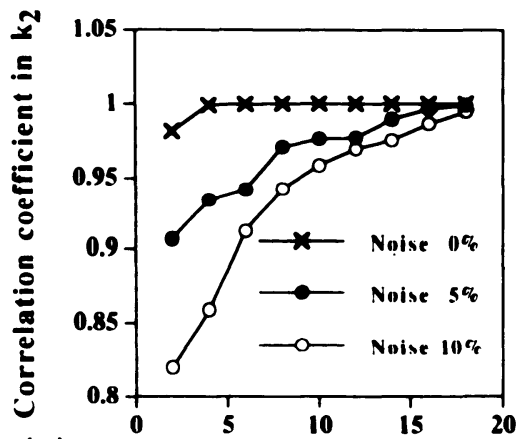

(c)

No. of hidden nodes

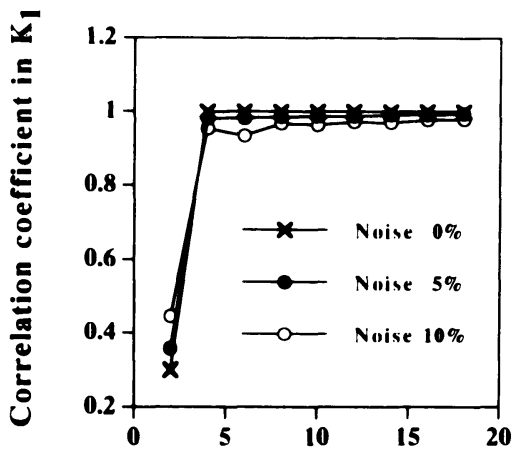

(b)

No. of hidden nodes

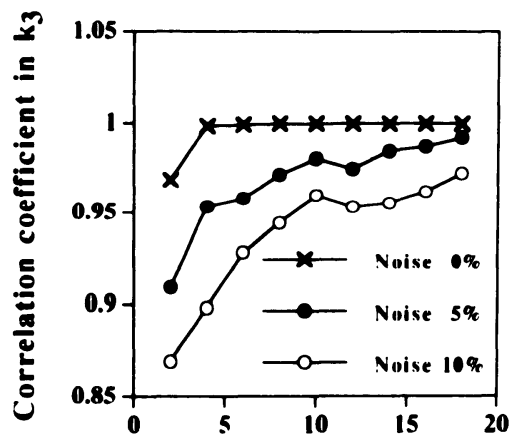

(d)

No. of hidden nodes

Fig. 3 (a) Relationship between the summation of squared error (SSE) and the number of hidden nodes in ANNs.

(b) Relationship between the correlation coefficient between the true and estimated $K_{1}$ values and the number of hidden nodes in ANNs.

(c) Relationship between the correlation coefficient between the true and estimated $k_{2}$ values and the number of hidden nodes in ANNs.

(d) Relationship between the correlation coefficient between the true and estimated $k_{3}$ values and the number of hidden nodes in ANNs.

In these cases, the number of training samples was taken as 500 .

(a) shows the relationship between SSE and the number of hidden nodes, and Figs. 3 (b), (c), and (d) show the correlation coefficients between the true and estimated values of $K_{1}(\mathrm{~b}), k_{2}(\mathrm{c})$, and $k_{3}$ (d) in the 500 training samples, as a function of the number of hidden nodes. An increase in the number of hidden nodes generally resulted in better performance.

Figure 4 (a) shows the relationship between the cpu time per iteration and the number of hidden nodes in the case when the number of training samples was fixed to 500, while Fig. 4 (b) shows the relationship between the cpu time per iteration and the number of training samples in the case when the number of hidden nodes was fixed to 10 . The cpu time increased in proportion to (the number of hidden nodes) $)^{1.713}$ and linearly with the number of training samples.

Table 1 summarizes the RMSE between the true and estimated values of $K_{1}, k_{2}, k_{3}$, and $K$ using ANNs for three noise levels ((a) for 0\%, (b) for 5\%, and (c) for 10\%). The results obtained using the NLSQ method are also shown for comparison. When there is no noise, the RMSEs in ANNs were significantly larger than those in the NLSQ method in all cases. When the noise 


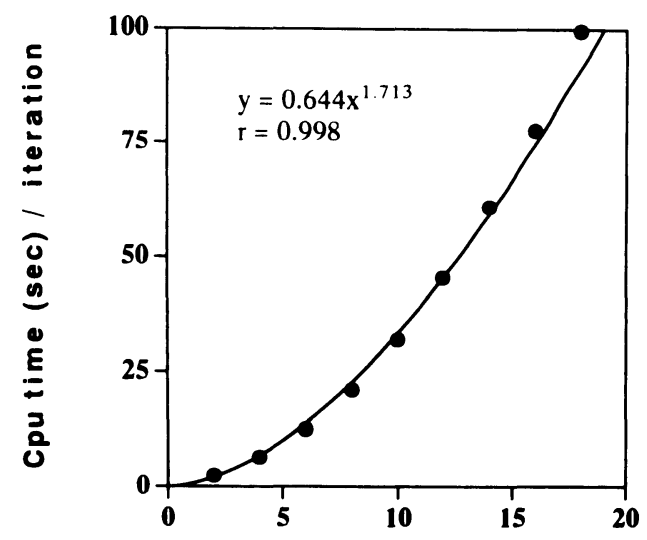

(a)

No. of hidden nodes

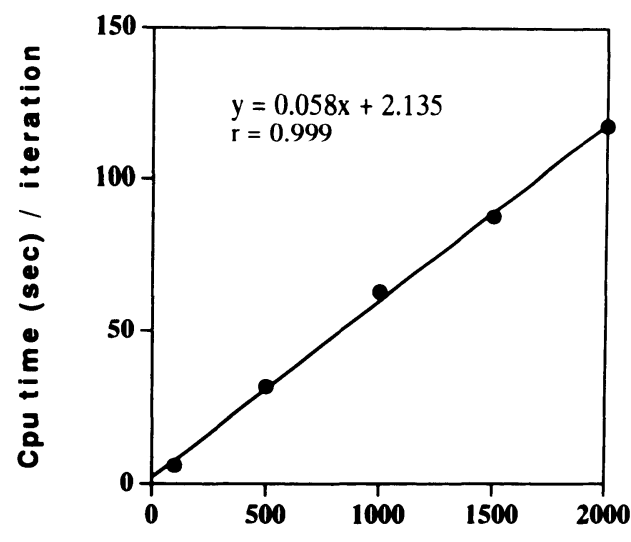

(b)

No. of training samples

Fig. 4 (a) Relationship between the computation (cpu) time per iteration and the number of hidden nodes. In this case, the number of training samples was fixed to 500 .

(b) Relationship between the cpu time per iteration and the number of training samples. In this case, the number of hidden nodes was fixed to 10 .

level is $5 \%$, there was no significant difference between ANNs with 1000 or 1500 training samples and the NLSQ method in $K_{1}, k_{2}$, and $k_{3}$. Except for these cases, the RMSEs in ANNs were significantly larger than those in the NLSQ method. On the other hand, when the noise level is 10\%, the RMSEs in ANNs were significantly smaller than those in the NLSQ method or there was no significant difference between them for $K_{1}, k_{2}$, and $k_{3}$ in all cases except for the case with 100 training samples. In the case of $K$, the RMSEs in ANNs were always significantly larger than those in the NLSQ method.

Table 2 summarizes the correlation coefficients between the true and estimated values of $K_{1}, k_{2}$, $k_{3}$, and $K$ using ANNs for three noise levels ((a) for $0 \%$, (b) for $5 \%$, and (c) for $10 \%$ ). As in Table 1, the results obtained using the NLSQ method are also shown. The tendency of the difference in the correlation coefficients between the two methods are almost the same as that in RMSE except for several cases. When the noise level is $10 \%$, the difference in the correlation coefficients in $K$ between the two methods was not significant in all cases of the number of training samples.

\section{Discussion}

ANNs with a layered structure as illustrated in Fig. 1 learn the relationship between input and output data from training samples presented repeatedly. The major factors affecting the performance of ANNs are the number of hidden layers, the number of hidden nodes within each layer and the number of training samples and iterations. Although some theoretical guidelines for optimizing these parameters with respect to a particular application can be found in the literature ${ }^{1), 17), 18)}$, this type of optimization is still somewhat of an open question. Probably, optimal network parameters are best determined through trial and error. It is generally advisable to use as simple an architecture as possible. For this reason, we selected the number of hidden layers as unity in the present study. In addition to the number of training samples, the performance of the ANNs depends on the variety of the training samples 
Table 1(a) Rood mean square errors (\%) between the true and estimated values in the case when the noise level is $0(\%)$

\begin{tabular}{ccccc}
\hline \multirow{2}{*}{$\begin{array}{c}\text { Number of } \\
\text { training samples }\end{array}$} & \multicolumn{4}{c}{ Root mean square error (\%) } \\
\cline { 2 - 5 } & $K_{1}$ & $k_{2}$ & $k_{3}$ & $K$ \\
\hline 100 & $1.55 \pm 0.80^{* *}$ & $6.73 \pm 8.29^{* *}$ & $9.25 \pm 4.11^{* *}$ & $6.40 \pm 3.03^{* *}$ \\
500 & $0.24 \pm 0.24^{* *}$ & $0.36 \pm 0.26^{* *}$ & $2.10 \pm 2.69^{* *}$ & $2.03 \pm 2.47^{* *}$ \\
1000 & $0.13 \pm 0.10^{* *}$ & $0.49 \pm 0.83^{* *}$ & $0.56 \pm 0.24^{* *}$ & $0.40 \pm 0.18^{* *}$ \\
1500 & $0.13 \pm 0.06^{* *}$ & $0.34 \pm 0.33^{* *}$ & $0.38 \pm 0.15^{* *}$ & $0.28 \pm 0.07^{* *}$ \\
\hline \multirow{2}{*}{ NLSQ method } & $6.58 \times 10^{-9} \pm$ & $1.84 \times 10^{-8} \pm$ & $1.13 \times 10^{-8} \pm$ & $3.61 \times 10^{-9} \pm$ \\
& $1.72 \times 10^{-9}$ & $4.84 \times 10^{-9}$ & $2.19 \times 10^{-9}$ & $2.96 \times 10^{-9}$ \\
\hline
\end{tabular}

Number in the table represents mean \pm standard deviation for ten cases.

Each case consists of 100 test samples.

NLSQ in the table stands for nonlinear leastsquares.

* Significantly $(\mathrm{p}<0.05)$ smaller compared to the NLSQ method.

** Significantly $(\mathrm{p}<0.05)$ larger compared to the NLSQ method.

Table 1(b) Rood mean square errors (\%) between the true and estimated values in the case when the noise level is $5(\%)$

\begin{tabular}{ccccr}
\hline \multirow{2}{*}{$\begin{array}{c}\text { Number of } \\
\text { training samples }\end{array}$} & \multicolumn{4}{c}{ Root mean square error (\%) } \\
\cline { 2 - 5 } & $K_{1}$ & $k_{2}$ & $k_{3}$ & $K$ \\
\hline 100 & $22.01 \pm 2.02^{* *}$ & $118.35 \pm 75.19^{* *}$ & $42.75 \pm 2.77^{* *}$ & $18.87 \pm 1.01^{* *}$ \\
500 & $5.93 \pm 0.46^{* *}$ & $19.53 \pm 2.31^{* *}$ & $15.60 \pm 2.51^{* *}$ & $7.39 \pm 2.70^{* *}$ \\
1000 & $5.50 \pm 0.59$ & $18.52 \pm 2.48$ & $14.54 \pm 2.40$ & $6.64 \pm 2.15^{* *}$ \\
1500 & $4.77 \pm 0.43$ & $18.69 \pm 2.16$ & $14.50 \pm 3.20$ & $5.74 \pm 2.81^{* *}$ \\
\hline \multirow{2}{*}{ NLSQ method } & $5.05 \pm 0.46$ & $17.00 \pm 2.53$ & $12.82 \pm 2.19$ & $2.88 \pm 1.92$ \\
\hline
\end{tabular}

Number in the table represents mean \pm standard deviation for ten cases.

Each case consists of 100 test samples.

NLSQ in the table stands for nonlinear leastsquares.

* Significantly $(\mathrm{p}<0.05)$ smaller compared to the NLSQ method.

** Significantly $(\mathrm{p}<0.05)$ larger compared to the NLSQ method.

Table 1(c) Rood mean square errors (\%) between the true and estimated values in the case when the noise level is $10(\%)$

\begin{tabular}{ccccc}
\hline \multirow{2}{*}{$\begin{array}{c}\text { Number of } \\
\text { training samples }\end{array}$} & \multicolumn{4}{c}{ Root mean square error (\%) } \\
\cline { 2 - 5 } & $K_{1}$ & $k_{2}$ & $k_{3}$ & $K$ \\
\hline 100 & $25.78 \pm 2.23^{* *}$ & $94.63 \pm 13.96^{* *}$ & $52.83 \pm 9.94^{* *}$ & $10.57 \pm 3.47^{* *}$ \\
500 & $11.14 \pm 2.44$ & $30.36 \pm 5.64^{*}$ & $24.37 \pm 5.67$ & $13.96 \pm 6.73^{* *}$ \\
1000 & $8.74 \pm 2.41^{*}$ & $26.89 \pm 7.86^{*}$ & $24.57 \pm 15.34$ & $10.69 \pm 4.88^{* *}$ \\
1500 & $6.84 \pm 0.56^{*}$ & $23.61 \pm 7.02^{*}$ & $20.46 \pm 5.02^{*}$ & $9.36 \pm 4.59^{* *}$ \\
\hline \multirow{2}{*}{ NLSQ method } & $10.45 \pm 0.99$ & $40.21 \pm 12.52$ & $29.31 \pm 10.48$ & $6.56 \pm 3.54$ \\
\hline
\end{tabular}

Number in the table represents mean \pm standard deviation for ten cases.

Each case consists of 100 test samples.

NLSQ in the table stands for nonlinear leastsquares.

* Significantly $(p<0.05)$ smaller compared to the NLSQ method.

** Significantly $(\mathrm{p}<0.05)$ larger compared to the NLSQ method. 
Table 2(a) Correlation coefficients between the true and estimated values in the case when the noise level is $0(\%)$

\begin{tabular}{ccccc}
\hline \multirow{2}{*}{$\begin{array}{c}\text { Number of } \\
\text { training samples }\end{array}$} & $K_{1}$ & $k_{2}$ & $k_{3}$ & $K$ \\
\cline { 2 - 5 } & $0.9982 \pm 0.0010^{* *}$ & $0.9962 \pm 0.0023^{* *}$ & $0.9890 \pm 0.0052^{* *}$ & $0.9920 \pm 0.0127^{* *}$ \\
500 & $0.9995 \pm 0.0014^{* *}$ & $0.9997 \pm 0.0006^{* *}$ & $0.9992 \pm 0.0013^{* *}$ & $0.9998 \pm 0.0001^{* *}$ \\
1000 & $1.0000 \pm 0.0000^{* *}$ & $1.0000 \pm 0.0001^{* *}$ & $0.9979 \pm 0.0045^{* *}$ & $0.9999 \pm 0.0001^{* *}$ \\
1500 & $1.0000 \pm 0.0000^{* *}$ & $1.0000 \pm 0.0000^{* *}$ & $1.0000 \pm 0.0000^{* *}$ & $1.0000 \pm 0.0000^{* *}$ \\
\hline \multirow{2}{*}{ NLSQ method } & $1.0000 \pm 0.0000$ & $1.0000 \pm 0.0000$ & $1.0000 \pm 0.0000$ & $1.0000 \pm 0.0000$ \\
\hline
\end{tabular}

Number in the table represents mean \pm standard deviation for ten cases.

Each case consists of 100 test samples. NLSQ in the table stands for nonlinear least-squares.

* Significantly $(\mathrm{p}<0.05)$ better compared to the NLSQ method.

** Significantly $(\mathrm{p}<0.05)$ worse compared to the NLSQ method.

Table 2(b) Correlation coefficients between the true and estimated values in the case when the noise level is $5(\%)$

\begin{tabular}{ccccc}
\hline \multirow{2}{*}{$\begin{array}{c}\text { Number of } \\
\text { training samples }\end{array}$} & \multicolumn{4}{c}{ Correlation coefficient } \\
\cline { 2 - 5 } & $K_{1}$ & $k_{2}$ & $k_{3}$ & $K$ \\
\hline 100 & $0.6924 \pm 0.0619^{* *}$ & $0.3241 \pm 0.1094^{* *}$ & $0.6065 \pm 0.0341^{* *}$ & $0.9036 \pm 0.0169^{* *}$ \\
500 & $0.9600 \pm 0.0068^{* *}$ & $0.8103 \pm 0.0319^{* *}$ & $0.8976 \pm 0.0251$ & $0.9863 \pm 0.0045^{* *}$ \\
1000 & $0.9623 \pm 0.0083^{* *}$ & $0.7850 \pm 0.0473^{* *}$ & $0.8882 \pm 0.0401$ & $0.9945 \pm 0.0011^{* *}$ \\
1500 & $0.9752 \pm 0.0044$ & $0.8498 \pm 0.0284$ & $0.9048 \pm 0.0211$ & $0.9952 \pm 0.0018^{* *}$ \\
\hline \multirow{2}{*}{ NLSQ method } & $0.9752 \pm 0.0026$ & $0.8793 \pm 0.0328$ & $0.8924 \pm 0.0546$ & $0.9980 \pm 0.0010$ \\
\hline
\end{tabular}

Number in the table represents mean \pm standard deviation for ten cases.

Each case consists of 100 test samples. NLSQ in the table stands for nonlinear least-squares.

* Significantly $(\mathrm{p}<0.05)$ better compared to the NLSQ method.

** Significantly $(\mathrm{p}<0.05)$ worse compared to the NLSQ method.

Table 2(c) Correlation coefficients between the true and estimated values in the case when the noise level is $10(\%)$

\begin{tabular}{ccccc}
\hline \multirow{2}{*}{$\begin{array}{c}\text { Number of } \\
\text { training samples }\end{array}$} & $K_{1}$ & $k_{2}$ & $k_{3}$ & $K$ \\
\cline { 2 - 5 } & $0.5413 \pm 0.0446^{* *}$ & $0.2043 \pm 0.0505^{* *}$ & $0.3817 \pm 0.089^{* *}$ & $0.9693 \pm 0.0092$ \\
100 & $0.8671 \pm 0.0286^{* *}$ & $0.6259 \pm 0.0789$ & $0.8177 \pm 0.0403^{*}$ & $0.9778 \pm 0.0068$ \\
500 & $0.9317 \pm 0.0155^{*}$ & $0.6993 \pm 0.0497$ & $0.8490 \pm 0.0256^{*}$ & $0.9847 \pm 0.0018$ \\
1000 & $0.9402 \pm 0.0096^{*}$ & $0.7909 \pm 0.0414^{*}$ & $0.8685 \pm 0.0293^{*}$ & $0.9898 \pm 0.0020$ \\
\hline \multirow{2}{*}{ NLSQ method } & $0.9043 \pm 0.0168$ & $0.6672 \pm 0.0747$ & $0.7215 \pm 0.0419$ & $0.8953 \pm 0.1944$ \\
\hline
\end{tabular}

Number in the table represents mean \pm standard deviation for ten cases.

Each case consists of 100 test samples. NLSQ in the table stands for nonlinear least-squares.

* Significantly $(\mathrm{p}<0.05)$ better compared to the NLSQ method.

** Significantly $(\mathrm{p}<0.05)$ worse compared to the NLSQ method. 
used. Ideally, the training data base should be created by selecting appropriate examples that present a wide distribution of all potential cases.

In the present simulation study, we used the 3-compartment FDG model ${ }^{4,5)}$ as an example, since it is the most popular and established kinetic model. As previously described, we generated training and test samples from the mean and SD values in the literature using Eq.(3). Therefore, the distribution of not only the training but also the test samples appears to be considerably wide, and these samples might include the data that could not exist in reality. This is considered to be the main reason why a relatively large number of training samples is necessary to get satisfactory results as shown in Tables 1 and 2.

As shown in Fig. 3, the performance of ANNs was generally better with increasing number of hidden nodes in the range between 2 and 18 . Furthermore, the performance of ANNs generally became better as the number of training samples increased as shown in Tables 1 and 2. In contrast, there was a report by Fisher et al. ${ }^{19)}$ that more input nodes, hidden nodes, and training iterations did not necessarily result in a better network. An important point concerning optimization of the number of nodes and the amount of training is that they will depend on the type and quantity of input data ${ }^{3)}$. Consequently, when ANNs are applied to any radiological problem, these parameters must be adjusted in a systematic manner. There are a few guidelines concerning this respect ${ }^{19}$. Iterations should be varied from approximately 10 to at least a few thousand. If the network is still performing well at high iterations, addition of more iterations should probably be attempted. Similarly, the number of hidden nodes should vary from approximately 2 to approximately 20. More than that would probably require an unrealistically large number of training cases to effectively train the network. Although each ANN was trained up to 1000 learning iterations or until SSE became less than $10^{-4}$ in the present study, approximately 500 iterations were sufficient because the change in SSE became little before 500 iterations in almost all the cases.

Several authors ${ }^{3), 19)}$ pointed out that with continued training, however, the networks made errors more frequently in the test cases. This occurred despite continued improvement in the error rate in the training cases. This phenomenon is a common observation in neural network analyses, and is referred to as "over-training" ${ }^{3), 19)}$. It is presumed to be due to memorization of the training cases by the network, that is, overtraining can cause a network to "memorize" its training set and degrade its performance on the testing set. However, such phenomenon was not observed as far as the studies performed here are concerned.

As shown in Tables 1 and 2, the accuracy of the parameter estimates seems to be worse or comparable to that obtained with the NLSQ method at lower noise levels. However, ANNs had a tendency to behave better than the NLSQ method at higher noise levels (Tables 1 and 2). Consequently, ANNs are considered to be less sensitive to the statistical noise included in the input data than the NLSQ method. This respect appears to be one of the potential advantages of using ANNs for kinetic parameter estimation.

The computational cost for training ANNs is generally very high. Although the performance of ANNs became better with an increase of the number of hidden nodes and training samples as previously described (Fig. 3, Tables 1 and 2), the cpu time per iteration also increased as shown in Fig. 4. However, once ANNs are trained adequately, they can estimate the kinetic parameters very fast, since the output is generated in a single pass without repeated iterations as in the NLSQ 
method $^{6}$ or without complicated procedures as in the weighted-integration method ${ }^{9}$. In addition to the noise tolerance mentioned above, this respect appears to be an important advantage of using ANNs for kinetic parameter estimation.

In the present study, we investigated the applicability of ANNs to kinetic parameter estimation only by computer simulation. The computerbased simulation method used here provides a convenient means to assess the performance of ANNs. The goal of the present study was to assess the effect of the number of hidden nodes, the number of training samples, and statistical noise. The computer-based simulation method enabled us to isolate these and to investigate a large number of study conditions, which could not be performed using real data. However, further investigations using real data are needed to establish the clinical usefulness of ANNs. These studies are currently in preparation. Furthermore, the plasma time-activity data was not used as an input data in ANNs as previously described. This corresponds to simulating the case when a standardized input function ${ }^{16)}$ was used. When dealing with individual input functions, the plasma time-activity data should also be used as an input data for training ANNs. This can be performed with minor modification. The effect of the errors contained in the input function on an ANN performance should also be investigated. This study is also currently in preparation.

In conclusion, our results suggest that an ANN approach may be an effective strategy for kinetic parameter estimation especially when generating pixel-by-pixel parametric images of kinetic rate constants.

\section{References}

1) Lippmann, R. P.:IEEE ASSP Mag., 4, 4-22 (1987)
2) Widrow, B., Winter, R. G. and Baxter, R. A. : IEEE Trans. Acoust. Speech Signal Processing, 36, 1109-1117 (1988)

3) Miller, A. S., Blott, B. H. and Hames, T. K. Med. Biol. Eng. Comput., 30, 449-464 (1992)

4) Phelps, M. E., Huang, S. C., Hoffman, E. J., Selin, C., Sokoloff, L. and Kuhl, D. E. : Ann. Neurol., 6, 371-388 (1979)

5) Huang, S. C., Phelps, M. E., Hoffman, E. J., Sideris, K., Selin, C. J. and Kuhl, D. E. : Am. J. Physiol., 238, E69-E82 (1980)

6) Beck, J. V. and Arnold, K. J. : "Parameter Estimation in Engineering and Science", John Wiley \& Sons, New York (1977)

7) Gjedde, A. : Brain Res. Rev., 4, 237-274 (1982)

8) Patlak, C. S., Blasberg, R. G. and Fenstermacher, J. D. : J. Cereb. Blood Flow Metab., 3, 1-7 (1983)

9) Alpert, N. M., Eriksson, L., Chang, J. Y., Berstrom, M., Litton, J. E., Correia, J. A., Bohm, C., AcKerman, R. H. and Taveras, J. M. : ibid., 4, 28-34 (1984)

10) Rumelhart, D. E., Hinton, G. E. and Williams, R. J. : Nature, 323, 533-536 (1986)

11) Scales, L. E. : "Introduction to Non-Linear Optimization”, Springer-Verlag, New York (1985)

12) Kuwabara, H., Evans, A. C. and Gjedde, A. : J. Cereb. Blood Flow Metab., 10, 180-189 (1990)

13) Reivich, M., Kuhl, D., Wolf, A., Greenberg, J., Phelps, M. E., Ido, T., Casella, V., Fowler, J., Hoffman, E. J., Alavi, A., Som, P. and Sokoloff, L. : Circ. Res., 44, 127-137 (1979)

14) Hawkins, R. A., Mans, A. M., Davis, D. W., Hibbard, L. S. and Lu, D. M. : J. Neurochem., 40, 1013-1018 (1983)

15) Evans, A. C., Diksic, M., Yamamoto, Y. L., Kato, A., Dagher, A., Redies, C. and Hakim, A. : J. Cereb. Blood Flow Metab., 6, 724-738 (1986)

16) Onishi, Y., Yonekura, Y., Nishizawa, S., Tanaka, F., Okazawa, H., Ishizu, K., Fujita, T., Konishi, J. and Mukai, T.:J. Nucl. Med., 37, 374-378 (1996)

17) Mirchandani, G. : IEEE Trans. Circuits and Systems, 36, 661-664 (1989)

18) Mehrotra, K. G., Mohan, C. K. and Ranka, S. : IEEE Trans. Neural Networks, 2, 548-558 (1991)

19) Fisher, R. E., Scott, J. A. and Palmer, E. L. : Radiology, 198, 699-706 (1996) 
要旨

\author{
人エニューラルネットワークを用いた動態パラメータの \\ 推定に関するシミュレーションによる検討 \\ 村瀬研也，棚田修二，池添潤平 \\ 愛媛大学医学部放射線医学教室 \\ 791-02 愛媛県温泉郡重信町志津川
}

\begin{abstract}
人工ニューラルネットワーク（ANN）の動態パラメータの推定への応用の可能性についてシ
\end{abstract} ミュレーションにより検討した。Sokoloff の糖代謝モデルと文献より得た速度定数の平均および 標準偏差の值を用いて, 種々の時間放射能曲線（TAC）を作成した。作成した TAC を入力とし, 用いた速度定数の值を出力として誤差逆伝搬法を用いて, 入力首, 中間層, 出力首からなる階層型 ニューラルネットワークの学習を行った。ANN の学習能は中間層のニューロンの数および学習 データの数の增加とともに向上した。ANN のパラメータの推定精度は, ノイズレベルが低い場合 には非線形最小二乗法に比べて劣っていたが, ノイズレベルが高い場合には非線形最小二乗法に比 べ概して優れていた。ANN は学習に長時間を要するが，一度学習を終えると高速にパラメー夕を 推定し得た。本検討により, ANN の動態パラメータの推定への応用の可能性が示唆された。 